\title{
Christophe Batsch, Mǎdǎlina Vârtejanu-Joubert (Ed.), Manières de penser dans l'Antiquité méditerranéenne et orientale
}

Mélanges offerts à Francis Schmidt par ses élèves, ses collègues et ses amis

Leiden, Brill, coll. « Supplements to the Journal for the Study of Judaism », vol. 134, 2009, 294 p.

Anna van den Kerchove

\section{(2) OpenEdition}

\section{Édition électronique}

URL : http://journals.openedition.org/assr/25168

DOI : $10.4000 /$ assr.25168

ISSN : $1777-5825$

Éditeur

Éditions de l'EHESS

\section{Édition imprimée}

Date de publication : 30 décembre 2013

Pagination : 135

ISSN : 0335-5985

\section{Référence électronique}

Anna van den Kerchove, « Christophe Batsch, Mǎdǎlina Vârtejanu-Joubert (Ed.), Manières de penser dans l'Antiquité méditerranéenne et orientale », Archives de sciences sociales des religions [En ligne], 164 | 2013, mis en ligne le 18 février 2014, consulté le 21 septembre 2020. URL : http:// journals.openedition.org/assr/25168; DOI : https://doi.org/10.4000/assr.25168 


\section{Christophe Batsch, Mădălina} Vârtejanu-Joubert (Ed.), Manières de penser dans l'Antiquité méditerranéenne et orientale

Mélanges offerts à Francis Schmidt par ses élèves, ses collègues et ses amis

Leiden, Brill, coll. « Supplements to the Journal for the Study of Judaism », vol. 134, 2009, 294 p.

Anna van den Kerchove

\section{RÉFÉRENCE}

Christophe Batsch, Mǎdǎlina Vârtejanu-Joubert (Ed.), Manières de penser dans l'Antiquité méditerranéenne et orientale, Mélanges offerts à Francis Schmidt par ses élèves, ses collègues et ses amis, Leiden, Brill, coll. « Supplements to the Journal for the Study of Judaism », vol. 134, 2009, 294 p. 
1 C. Batsch et M. Vârtejanu-Joubert ont édité un beau volume d'hommage à Francis Schmidt, dont l'importance des travaux pour les études juives est rappelée dans un premier temps. En écho aux principaux centres d'intérêt de Francis Schmidt (historiographie, comparatisme et anthropologie historique), les éditeurs ont réuni quinze textes émanant de spécialistes de l'Antiquité, juive d'abord, mais aussi grecque, romaine, mésopotamienne, l'ensemble étant réparti en quatre parties : « Historiographie », « Rationalité et sciences», «Lien social, rites et identité », « Mentalités ».

2 La première partie comporte les contributions, stimulantes, des deux éditeurs. La première ("Théorie et

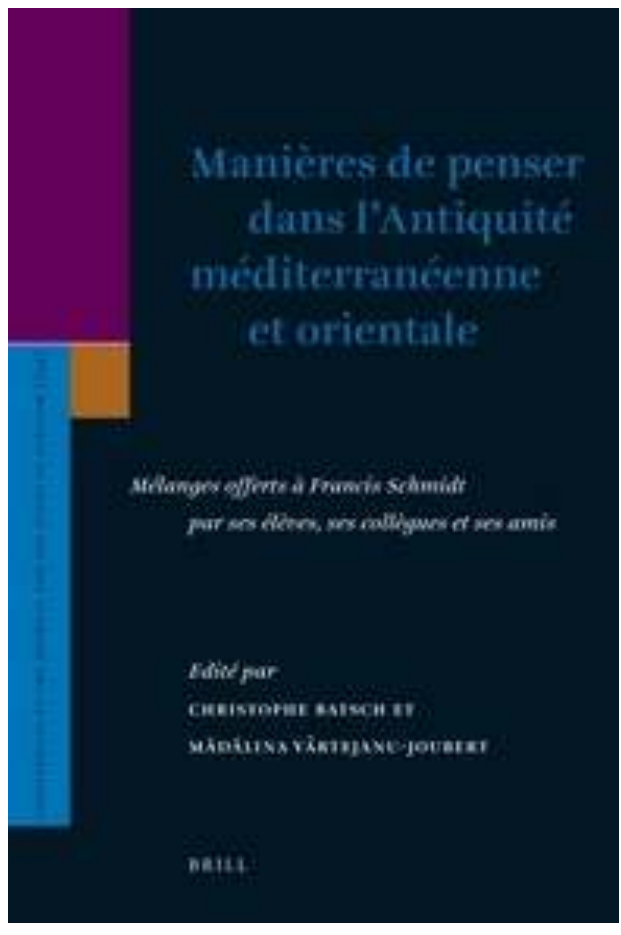
méthode d'une "laïcisation" de l'Écriture :

les sources bibliques dans le De iure belli ac pacis de Grotius ») peut surprendre, car elle concerne la Renaissance, le théologien Hugo de Groot et son De iure. En quelques pages lumineuses, Batsch montre toute la différence entre la manière de penser antique et celle qui se développe à partir de la Renaissance. L'évolution de l'attitude envers un texte sacré est symptomatique de cette différence. En effet, dans le domaine du droit, Hugo de Groot initie une utilisation d'un écrit sacré que Batsch qualifie (à juste titre à notre avis) de laïcisée. Ces pages, et la méthode de Hugo de Groot, mériteraient d'être méditées et intéresseront à la fois les antiquistes, les modernistes et les contemporanéistes.

3 Dans un autre domaine, avec «La mystique à Qumrân : regards historiographiques et déconstruction de la notion ", M. Vârtejanu-Joubert nous amène une nouvelle fois à nous interroger sur la pertinence des catégories heuristiques que beaucoup de chercheurs utilisent couramment en oubliant qu'au départ il s'agit d'hypothèses. À partir de Qumrân et d'une étude historiographique de la catégorie "mystique ", l'auteur invite chaque chercheur à remettre en cause ces catégories (devenues des piliers) en fonction de l'évolution de la recherche, à s'interroger sur les aspects conceptuels et sur la manière différente dont les sources sont abordées en fonction de l'objet d'étude.

4 La deuxième partie s'ouvre par l'article de J.-J. Glassner, sur « L'herméneutique des devins mésopotamiens ». À partir d'une recherche sur la divination et sur la manière dont la mise par écrit modifie le régime de rationalité, l'auteur montre comment l'acte de l'écriture modifie la relation des ritualistes au rituel et comment il génère lui-même de la pensée. Les trois contributions suivantes abordent le judaïsme. A. Lemaire, dans Esséniens et Pharisiens : deux interprétations de l'Écriture ", livre une פשר et étude minutieuse du vocabulaire utilisé par les Esséniens et les Pharisiens pour qualifier leur exégèse; le vocabulaire légèrement différent témoigne de deux conceptions de l'interprétation des Écritures qu'il faut replacer dans le contexte de 
mise en forme du canon juif. D. Dimant, dans «On Righteous and Sinners: $4 \mathrm{Q} 181$ Reconsidered ", propose une édition, une traduction, un commentaire et des notes sur 4Q181. Attentive au moindre détail, elle cherche à mieux caractériser le lien entre ce document et 4Q180 ; elle conclut, contre l'avis de Jozef Milik, que 4Q181 cite $4 \mathrm{Q} 180$ ou une source commune et qu'en aucun cas il ne s'agit de deux versions d'un même document. Dans «Démétrius le Chronographe doit-il être regardé comme le père de l'historiographie qoumrânienne? », J.-C. Duls ouvre à nouveau le débat sur les rapports entre le calendrier de $Q$ et Démétrius le Chronographe et propose une nouvelle traduction du texte de ce dernier transmis par Eusèbe de Césarée. La deuxième partie se clôt avec la contribution intéressante d'A. Desreumaux, "Comment peut-on écrire en syriaque? Ou Des problèmes du scribe devant sa page blanche ». S'intéressant en propre à l'acte d'écrire et, plus particulièrement, à la disposition de la feuille et au ductus de l'écriture syriaque, A. Desreumaux en rappelle l'importance et met en avant le lien réciproque entre écriture et pensée.

5 La troisième partie s'ouvre par trois contributions sur le judaïsme. Celle d'A. Marx, «Ethnicité et pérennité de l'Israël antique. Les stratégies identitaires consécutives à la disparition du royaume de Juda" s'inscrit dans les recherches sur l'identité et sa construction. À partir d'une étude sur les conséquences de la fin du royaume de Juda, l'auteur montre comment l'identité est pensée pour donner à la fois un sens au passé (la catastrophe) et au futur. Avec "Purity of Lineage in Talmudic Babylonia", A. Oppenheimer s'intéresse à la manière dont le Talmud de Babylone envisage la pureté de lignage en lien avec la géographie. Dans "The Celebration of the Passover in GraecoRoman Alexandria ", N. de Lange montre que la célébration de la Pâque est identique partout et il compare ce qu'en dit Philon d'Alexandrie avec le Pentateuque grec. La contribution suivante aborde le Nouveau Testament. C. Grappe, dans «Prolongements et subversions de la pensée du Temple dans le Nouveau Testament au miroir de l'action et de la prédication de Jésus dans l'Évangile selon Marc », prolonge sa recherche sur la pensée du Temple, dans le Nouveau Testament. La troisième partie se clôt par deux contributions sur le monde romain. Avec "Les pontifes romains et le parjure", $\mathrm{J}$. Scheid s'intéresse à un thème peu abordé du droit romain, celui du parjure, une procédure qui relève de la responsabilité des pontifes et qui est peu documentée. La lecture conjointe de Plaute et d'Horace permet d'obtenir quelques informations supplémentaires. La contribution de N. Belayche " "Un dieu est né..." à Stratonicée de Carie (I Stratonikeia 10) » s'inscrit dans les recherches de l'auteur sur la représentation du divin; à partir d'un document relatant l'apparition d'une nouvelle divinité à Stratonicée de Carie, Zeus Panamaros, elle s'interroge sur la naissance cultuelle d'une divinité et sur la construction historique de la représentation d'un dieu dans une cité.

6 La quatrième et dernière partie de l'ouvrage débute par un article de P. Lanfranchi, «Entre construction liturgique et polémique antijuive. La collection de bénédictions d'origine juive des Constitutions Apostoliques ». Plusieurs textes de la liturgie synagogale ne sont désormais documentés que grâce à des écrits chrétiens. L'auteur s'intéresse ainsi à la façon dont des bénédictions juives ont été intégrées dans les Constitutions Apostoliques, A Comprendre Dans Le Cadre D'une Concurrence Entre Bénédictions Juives Et Bénédictions Chrétiennes. M. Philonenko, Avec « "La Lumière Dans Mon Cœur Vient De Ses Mystères Merveilleux". De La Règle De La Communauté XI 5 A II Corinthiens 4,5 (Contribution A L'étude Du Sociolecte Esséno-Qoumrânien) », Prolonge Sa Recherche Sur Le Sociolecte Esséno-Qoumrânien, En Relevant Les Parallèles De Deux Tournures Linguistiques, A La Fois Dans La Règle De La Communauté Et Dans D'autres 
Ecrits Juifs. Comme A Son Habitude, L'auteur Fournit Au Lecteur Un Grand Nombre De Citations, Intéressantes Par Elles-Mêmes, Mais Qui Ne Sont Pas Contextualisées Et Dont Le Rapprochement N'est Pas Toujours Argumenté. Les Deux Dernières Contributions S'intéressent Aux Mondes Grec Et Romain. R. Koch-Piettre, Dans «Oreste, Un Héros Grec Dans La Religion Romaine ", S'intéresse A La Figure d'Oreste Sur Le Sol Latin. S. Crippa Poursuit Ses Recherches Sur Le Langage Et Le Sacré Dans «Les Marges Du Langage Dans Les Contextes Sacrés : $\Phi \theta o ́ \gamma \gamma o \sigma, \Phi \theta \varepsilon ́ \gamma \gamma \sigma \mu \alpha 1$ »; Elle Propose Une Etude De L'usage De Ces Termes Dans Les Ecrits Grecs (Philosophiques Et Rituelles), Pour Conclure Avec L'hypothèse Que Ces Vocables Ont Pu Constituer «Le Vocabulaire Technique Pour Désigner Les Phonations Présentes Dans Les Pratiques Sacrées ».

7 L'ouvrage se clôt avec un index des sources.

8 Bien que chaque article porte sur un sujet spécifique, leur lecture est d'un grand profit pour tout chercheur intéressé à l'histoire des idées, des mentalités et des religions. En effet, ils nourrissent sa réflexion méthodologique et épistémologique, en revenant en particulier sur la pertinence des catégories utilisées (non forcément pour les remettre systématiquement en cause, mais pour prendre conscience de leur caractère hypothétique), sur l'incidence du choix de l'objet étudié sur la manière de lire et de comprendre les sources et sur la philologie... 